\title{
LETTER FROM THE EDITOR IN CHIEF
}

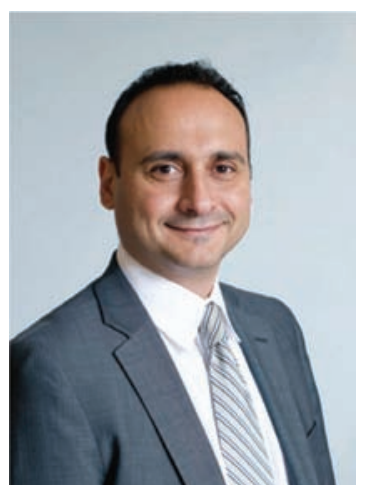

Dear Readers,

I would like to take this opportunity to reflect back on the major developments in the field of cardiac electrophysiology in 2016. The rate of growth that we observed over the past few years continued, and many areas of cardiac EP witnessed significant advances that have the potential to improve the outcome of patients with cardiac arrhythmia.

Catheter ablation for atrial fibrillation continued to grow rapidly. Contact force sensing and the second generation cryoballoon helped improve the success rate of pulmonary vein isolation for paroxysmal AF. The next frontier in this area is assessment of ablation lesion, and numerous promising technologies are currently being developed with the objective of accurately assessing optimal lesion formation. These technologies have the potential not only to further improve the success rate, but also reduce the risk of damage to collateral structures. The area of ablation for persistent AF has also seen major developments with most of the focus on rotor ablation and scar imaging and modification. Many high density mapping systems are currently being developed for rotor/driver mapping. Moreover, a multinational randomized clinical study comparing PVI alone vs. PVI plus atrial scar ablation for persistent AF is currently under way. Ventricular tachycardia ablation has also witnessed important advances including the expansion of clinical investigations of tools to allow the creation of deeper lesions such as bipolar ablation and needle electrode. Another area of rapid growth in 2016 is stroke prevention. The use of the Watchman ${ }^{\mathrm{TM}}$ device (Boston Scientific, Maple Grove, MN) continues to grow rapidly, and randomized clinical studies comparing it to the Amulet $^{\mathrm{TM}}$ (St. Jude Medical, Minnetonka, MN) and other devices have been initiated. In addition, other novel left atrial appendage closure tools are currently being tested in small clinical studies outside the United and are showing promise. The use of direct oral anticoagulants maintained a solid growth and continued to replace warfarin.

The field of device therapy has also witnessed important advances. Leadless pacemakers became commercially available in the US. The use of subcutaneous ICD is also expanding, and clinical studies testing the use of leadless pacing and subcutaneous ICD are being planned. On the resynchronization front, multisite pacing appears promising and its use expanding.

As for the state of the Journal, I am happy to report some important modifications. Two sections have been introduced: "Tracing of the Month" led by Dr. Kevin Heist, and "Images in Cardiac EP" led by Dr. Claudio Tonto. I hope that you will find these new sections educational and helpful to your practices. The Journal also continues to grow with respect to readership and submission of manuscripts.

I hope that you enjoy reading this issue. Best wishes for a happy and healthy New Year.

$$
\text { Regards, }
$$

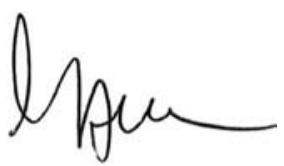

Moussa Mansour, MD, FHRS, FACC

Editor-in-Chief

The Journal of Innovations in Cardiac Rhythm Management

MMansour@InnovationsInCRM.com

Director, Cardiac Electrophysiology Laboratory

Director, Atrial Fibrillation Program

Massachusetts General Hospital Boston, MA 